\title{
Granular Computing
}

\author{
Collin Lee \\ Stanford University
}

\begin{abstract}
Granular computing is a new style of computing where applications are composed of large numbers (thousands to millions) of very short-lived $(10-100 \mu \mathrm{s})$ tasks. Today's systems and infrastructure were designed to support millisecondscale operations and are inadequate to meet the demands of granular computing. In this position paper we discuss the challenges of supporting granular applications, such as handling extreme bursts of activity, and we present a few initial ideas about the infrastructure required to enable granular computing, such as new mechanisms for communication and persistence.
\end{abstract}

CCS Concepts - Software and its engineering $\rightarrow$ Distributed systems organizing principles; Software infrastructure; Cloud computing; Software performance.

Keywords abstraction, burst, computing, datacenter, efficiency, granular, infrastructure, latency, software, task

\section{ACM Reference Format:}

Collin Lee and John Ousterhout. 2019. Granular Computing. In Workshop on Hot Topics in Operating Systems (HotOS '19), May 13-15, 2019, Bertinoro, Italy. ACM, New York, NY, USA, 6 pages. https://doi.org/10.1145/3317550.3321447

\section{Introduction}

In this position paper we propose the development of software infrastructure to support a new style of computation called granular computing (summarized in Table 1). In granular computing, applications are composed of very large numbers of small tasks running in large datacenter clusters. Individual tasks may execute for as little as a few microseconds. Applications can execute thousands to millions of tasks, both in parallel and sequentially, spread across thousands of machines. In addition, we expect granular applications to be bursty, harnessing thousands of machines for just a few intense milliseconds of computation. We believe that granular computing will enable new kinds of applications that cannot exist today, such as those that analyze large amounts of irregular data in real time.

Permission to make digital or hard copies of part or all of this work for personal or classroom use is granted without fee provided that copies are not made or distributed for profit or commercial advantage and that copies bear this notice and the full citation on the first page. Copyrights for thirdparty components of this work must be honored. For all other uses, contact the owner/author(s).

HotOS '19, May 13-15, 2019, Bertinoro, Italy

(C) 2019 Copyright held by the owner/author(s).

ACM ISBN 978-1-4503-6727-1/19/05.

https://doi.org/10.1145/3317550.3321447
John Ousterhout

Stanford University

\begin{tabular}{cl}
\hline Property & Scale \\
\hline Small Tasks & $10-100$ microseconds \\
Large Scale & $1 \mathrm{k}-1 \mathrm{M}$ cooperating tasks \\
Short Bursts & $1-10$ milliseconds of activity \\
\hline Table 1. Properties of Granular Computing
\end{tabular}

Today's software systems cannot support granular applications. System overheads are too high to support small tasks efficiently or to scale up and down rapidly. Granular computing requires software infrastructure that operates at microsecond scale, but today's systems are designed for millisecond scale [2]. Thus, granular computing will require us to rethink much of the infrastructure that runs below the level of applications, as well as the basic structure of the applications themselves.

There is already a movement afoot towards more granular applications. For example, function-as-a-service platforms like AWS Lambda [1] support tasks as small as 100 milliseconds, cluster orchestrators such as Kubernetes [17] can respond to load changes in a few seconds, and other approaches have been proposed [11]. Rather than making incremental improvements to these systems, we propose taking the notion of granularity to its extreme, addressing the following questions:

- What are the smallest tasks and largest bursts that can be supported efficiently at datacenter scale on today's hardware?

- What are the limits that prevent even smaller tasks and larger bursts from running efficiently?

- What abstractions, architectures, and algorithms enable the smallest possible tasks and the largest possible bursts?

This extreme approach will advance the frontier of knowledge more rapidly than an incremental approach, and it will stimulate more innovative design thinking.

The remainder of this position paper discusses how granular computing might be useful (Section 2), why existing systems cannot support it (Section 3), and what infrastructure for granular computing might look like (Section 4).

\section{Motivation: New Applications}

If an efficient platform for granular computing were available, we hypothesize that it would enable exciting new applications that are difficult or impossible to create today. It is hard to point to existing applications as examples, but this is unsurprising since there is currently no platform that supports 
such applications. In this section we speculate about two classes of applications that might be enabled by granular computing.

\subsection{Real-Time Data-Intensive Processing}

Today it is difficult to create applications that analyze large amounts of data and return results in real time. Big Data analytics tools such as MapReduce [5] and Spark [18] can process huge datasets, but their execution times typically range from minutes to hours. Systems with interactive response times must severely limit the amount of data they consider in each query. For example, services such as Facebook must respond to incoming HTTP requests in a few hundred milliseconds. In order to meet this requirement, at most a few thousand pieces of data can be accessed while servicing each request (the time for a single data access ranges from a few hundred microseconds to a few milliseconds, so there is enough time for at most a few hundred sequential accesses; another factor of around 5-10x can be achieved by issuing requests concurrently). The functionality of online applications like these is limited by the amount of data they can consider.

Granular computing has two features that will allow more data to be processed in less time. First, it will reduce the latency overhead for invoking requests, so more sequential requests can be completed within a given time budget. Second, granular computing will allow concurrency to be harnessed more efficiently (e.g. by reducing the time to initiate a burst involving hundreds or thousands of nodes); this will enable a greater degree of concurrency than is possible today. As an example, if the time for an individual data access is reduced by $100 \mathrm{x}$ and the degree of concurrency can be increased by a factor of $100 x$, then $10000 x$ more data can be accessed without increasing the user-visible response time.

\subsection{Micro-Lambdas}

Cloud computing vendors have recently introduced functionas-a-service or "serverless" platforms, such as AWS Lambda [1] and Azure Functions [9], which allow applications to execute individual functions such as HTTP requests. Applications are billed only for the resources required to execute their requests. Today, the minimum unit of billing is 100 milliseconds for AWS Lambda and 1 second for Azure Functions. Additionally, the invocation latency overheads range from tens of milliseconds to seconds. As such, these platforms are neither cost-effective nor resource efficient for functions that only execute for a few milliseconds. Granular computing will support tasks three orders of magnitude smaller, thereby making serverless computing cost-effective for a broader range of applications. In addition, systems such as AWS Lambda do not support communication between lambdas; granular computing will allow lambdas to work together in large-scale bursts.

\section{Challenges}

The basic properties of granular computing, extremely short tasks and extremely intense bursts, create significant challenges for system software. This section discusses the challenges and the reasons why current software infrastructure cannot meet them.

Low Latency. Granular computing will require extremely low latency in several areas, including task initiation and network communication. If a task executes for only $10 \mu \mathrm{s}$, then task invocation must not take more than $1 \mu$ s or so; if a burst of 1000 tasks executes for only $1 \mathrm{~ms}$, then it must not take more than $100 \mu$ s or so to initiate all of the tasks in the burst. Microsecond-scale network communication is essential, both to support fast burst startup and to communicate between short-lived tasks. Furthermore, the latency that matters is usually tail latency, not median [4]; tail latency is particularly difficult to optimize because it requires eliminating even infrequent sources of overhead, such as congestion.

Extreme Bursts. Bursts that are large-scale and short-lived introduce two challenges. The first challenge is how to start such bursts very quickly (on the order of $100 \mu$ s); this will require communication with many servers in parallel (sequential communication would take far too long) and allocation of resources on each of them. The second challenge with bursts is resource utilization. By definition, a bursty application does not make efficient use of its resources: it requires a large resource allocation during bursts, but it does not use the resources between bursts. In order to use resources efficiently, non-bursty background tasks must be run during the lulls between bursts, and the system must be able to preempt them very quickly at the start of the next burst.

Today's software stacks have overheads that are far too high to support granular computing. For example:

- Creating a thread on Linux takes about $10 \mu \mathrm{s}$.

- A network round-trip between two servers in the same datacenter can take $500 \mu$ s or more when the network is loaded.

- Spinning up a Linux container takes hundreds of milliseconds.

- Initiating a job that spans a few hundred nodes with Spark [18], Kubernetes [17], or AWS Lambda [1] can take one second or more.

Several factors contribute to today's high overheads. First, existing software infrastructure was designed to operate at a timescale of milliseconds, not microseconds [2]. In particular, the assumption of disk-based storage permeates much of the design of today's software. If most operations eventually result in a disk $\mathrm{I} / \mathrm{O}$ requiring 5-10 ms, then overheads of tens or even hundreds of microseconds are insignificant. However, overheads of this scale are show-stoppers for granular computing; unfortunately, they have become common. 
A second problem with existing software systems is that they have become highly layered. Layering is a valuable tool for managing complexity, but each layer crossing adds overhead. Latency accumulates across a large number of layers, with no single place that can be optimized to improve performance. New software layers are constantly being introduced to improve manageability or to virtualize resources; one recent example is new layers for network virtualization [3, 12]. Granular computing will require a significant re-architecture of the software stack to eliminate layers or bypass them in the common case.

Because of these overheads, systems are often optimized for throughput at the expense of latency. This allows them to amortize high startup costs across large blocks of data, but it typically makes latency even worse. For example, collecting data into batches usually improves throughput, but it increases the latency for individual operations.

It seems unlikely that the problems with today's system software can be solved with a few localized changes. Significant changes both in architecture and implementation will be required to support granular computing.

\section{Granular Computing Infrastructure}

Enabling granular applications will require new infrastructure that meets the challenges of granular computing; we will refer to any infrastructure that supports granular computing as granular computing infrastructure or granular infrastructure. Building out infrastructure for granular computing will require rethinking the datacenter software stack and developing new platforms, services, and frameworks. In this section we highlight some of the specific issues that implementations of granular infrastructure will need to address and suggest possible solutions for these issues. We don't expect to cover every important issue nor do we expect the proposed designs necessarily to be the final answer. Instead, we hope this will generate discussion around how to develop the infrastructure necessary to support granular computing.

The most obvious goals for any granular computing infrastructure center around efficiency. For example, granular infrastructure implementations must provide the high performance necessary to support the small task sizes and extreme bursts of granular applications. In addition, the infrastructure must ensure that datacenter resources are fully utilized, in spite of the low duty cycles of bursty applications.

An interesting question is whether significant new abstractions are needed for granular computing: if we just make existing abstractions faster, is that good enough? We believe that new abstractions will be required for two main reasons. First, new abstractions can simplify application development by solving problems that are common across granular applications. Big Data frameworks such as MapReduce [5] and Spark [18] introduced new abstractions such as resilient distributed datasets in Spark, which made applications easier to write; we expect new abstractions to arise for granular computing as well.

Second, we believe that new abstractions will play an important role in achieving high performance. One of the reasons for the high overheads of today's system software is the large number of layers. Raising the level of the abstraction for application development allows more work to be done in the infrastructure layer; this creates opportunities for eliminating layers or restructuring them to maximize performance.

Abstractions will exist at many levels of a granular computing infrastructure stack. At the highest levels, application development frameworks will provide specialized abstractions for a variety of different types of granular applications, similar to how Spark [18] and Pregel [8] support different styles of computation for Big Data applications. At lower levels, the infrastructure can provide abstractions for computation, communication, and storage that fit the needs of granular applications more generally. In this paper, we have chosen to focus on the lower level aspects of the infrastructure that will provide the core facilities for all granular computing applications.

\subsection{Services}

One of the ways that the infrastructure can raise the level of abstraction for granular applications is to take responsibility for distributed resource management. Distributed applications today must be aware of exactly which machines they are using and must handle the changes to their physical footprint in response to machines crashing and loads fluctuating. This awareness of the physical machine would become much more challenging to handle in the drastically more dynamic, bursty, and sharded environment of granular computing. Instead, we propose that information about physical locations should be encapsulated in the infrastructure: applications should not be aware of which specific machines they are using, or even how many distinct machines they are using.

We use the term service to describe the basic unit of computation in granular computing. A service is a logically distinct application component that performs work based on invocations of an API. Services are not tied to particular machines or processes; a service may utilize multiple cores on multiple servers and its structure may change dynamically. A service requests resources such as computation and storage, and the granular infrastructure decides which servers should provide those resources. For some resources, such as computation, the infrastructure can automatically detect when resource usage should be scaled up or down; as much as possible, it should handle scaling automatically. In other cases, such as storage, the infrastructure can help with scaling but the service will also need to help (e.g. by identifying shards that can be located on different machines).

We propose that granular infrastructure designs should explicitly distinguish between two types of services: stateless 
and stateful. We distinguish between the two because they have different properties that can be leveraged to manage them more efficiently. Stateless services are similar to lambdas in AWS: they retain no state between invocations of their API; any data needed for the request is either provided as a parameter or fetched from a stateful service. Web front-ends and data analytics engines are typically stateless. In contrast, stateful services maintain data across API invocations. General purpose storage systems and databases are examples of stateful services.

Stateless services are generally easier to manage and allow for more dynamic management approaches. An invocation of a stateless service can be processed on any core that has access to the service's code. This makes it possible (and desirable) for the infrastructure to perform automatic scaling (adjusting the number of cores used by the service to match its load) and load balancing (choosing where each incoming request will be handled in order to balance the load across the available resources). Crash recovery is trivial for stateless services, since there is no service data to recover.

Stateful services are more constrained: an invocation must be serviced by a core that has access to particular data, such as a specific key in a key-value store. This limits the opportunities for load balancing. Furthermore, the infrastructure cannot automatically scale a stateful service, since scaling requires changes to the shard and/or replica structure of the data. Instead, scaling must be performed cooperatively with the service. Crash recovery is also more complex for stateful services, since the service may wish to recover lost data. Stateful services will require additional support from granular computing infrastructure, such as mechanisms for allocating storage and defining shards.

\subsection{Addressing}

Addressing is a common problem for traditional applications and granular applications alike. Applications require some form of addressing to specify the target of communication, such as when invoking a service's API. Most systems today use port-based addressing to identify and communicate with a deployed service. Port-based addressing, however, is too low-level and doesn't work well when the physical locations of services change dynamically in response to failures and load changes. To solve these problems today, each service must create its own application-specific addressing layer on top of port-based addressing.

To better support granular applications, granular infrastructure should provide new mechanisms for addressing that do not expose physical locations. This will eliminate the need for services to create their own virtual addressing layers. It will also raise the level of abstraction, which will allow the infrastructure to optimize its implementation (e.g. by combining the higher-level addressing mechanism with the basic transport mechanism).
We propose two kinds of addressing that will raise the level of abstraction: service-based addressing and resource-based addressing. In service-based addressing, communication is directed at a particular service, not a port, and the infrastructure can load-balance requests across the service's instances. Service-based addressing is most appropriate for stateless services where there is no distinction between service instances. In resource-based addressing, communication is directed at a particular resource, such as a piece of data with a particular key in a key-value store; the infrastructure coordinates with the service to find the appropriate service shard and directs the request to it. While data resources will be most common, we imagine other resources like pre-programmed FPGAs, GPUs, or TPUs could also be addressed. Resourcebased addressing makes sense for stateful services. In both service-based and resource-based addressing, the infrastructure will automatically handle service reconfigurations, even in the middle of a request.

\subsection{Group Communication}

Most network communication mechanisms today are designed for point-to-point communication with a single sender and single receiver. In contrast, we expect granular applications to depend heavily on group communication in which there is concurrent communication between multiple senders and receivers. Group communication is fundamental to harnessing concurrency. For example, a burst is typically triggered from a single starting point; the initiator must communicate with multiple workers to enlist them in the burst computation.

Most systems today do not have good support for group communication beyond some form of asynchronous RPC. RPCs, however, are not well suited for large groups or tight latency requirements. In our experience, even the fastest RPCs incur a few hundred nanoseconds of overhead per RPC. When bursting to thousands of tasks, this RPC overhead would add hundreds of microseconds of latency.

Granular computing infrastructure implementations will need to provide more efficient mechanisms for group communication by raising the level of abstraction from point-topoint RPCs to group-level operations. We also expect group communication to be closely related to the higher-level addressing mechanisms discussed in Section 4.2, such as "send this message to all instances of a service" or "send this message to all copies of a particular object." We expect the group communication mechanism to ensure reliable message delivery but have not yet seen the need for enforcing ordering between broadcasts like those used in some consensus systems [6].

There are systems today that provide some form of group communication, but they are not well suited for granular computing. The collective communication facilities defined in MPI [15], with operators such as "broadcast," "scatter," and "all-gather," provide perhaps the closest candidate to the 
needs of granular computing. However, the MPI operators use a form of addressing that is essentially port-based. Granular computing infrastructure implementations will require support for their higher-level addressing mechanisms. Furthermore, MPI is designed for application structures that are relatively static. Publish-subscribe systems [7, 13, 16] provide rich addressing models, but they are not well optimized for latency. For example, we measured a best case one-way latency for Kafka [7] of about 600 microseconds.

New implementations will need to be developed to efficiently support group communication with the higher-level addressing mechanisms proposed for granular computing.

\subsection{Handling Bursts}

Supporting bursty application will be extremely challenging both in terms of latency and in terms of efficient resource utilization. Here are a few issues and ideas around how granular infrastructure implementations might handle bursts.

Services running on granular computing infrastructure will need to be loaded well before invocation in order to achieve the microsecond scale invocation latencies needed by granular applications. Before a service can execute a single line of code, the host machine must already have the code and have it loaded into an execution context like a process, container, or VM. Starting a process or container can take as much as a few hundred milliseconds. Starting VMs and shipping code could take seconds to minutes. The startup cost of shipping code and launching a protected execution context is too high to be executed on demand as services are invoked. Instead, the infrastructure will likely need to have the service pre-deployed well in advance of the invocation so that the service can run as soon as it is invoked.

Services will likely need to stay loaded for long periods of time, which makes their idle resource consumption a key issue. Granular applications are bursty with low duty cycles but may require the full cluster when active. Under certain conditions, it may be necessary for every service to be preloaded on every machine. As a result, machines might have hundreds or thousands of idle services. Any resources held by an idle service become unavailable to executing applications, which reduces resource utilization. For example, if the code is loaded into main memory or a neural network inference model is loaded onto a GPU, these resources will be unavailable. We do not currently have good solutions for this problem.

Services with the smallest units of execution will also require new techniques for protection domains. Switching between Linux processes takes roughly $4 \mu$ s. For services with $10 \mu$ s tasks, this context switch cost is intolerable. One option would be to keep the context loaded. Unfortunately, this would cause the service to consume a CPU core even when idle. To reduce the cost of protection domain switches, granular infrastructure designs may need to consider other forms of protection such as language level protection or the revival of memory segmentation.

Finally, to achieve high resource utilization, the infrastructure must support resource sharing between bursty granular applications and batch-oriented background applications. Background applications, which run at longer timescales (minutes - hours), can run using the spare resources left unused by granular applications; they are not bothered by the periodic interruptions when granular application bursts occur. Recent work has shown that it is possible to effectively share resources with batch workloads without harming the latency of the latency-sensitive applications [10, 14]. However, in order to also maintain low invocation latencies, granular infrastructure implementations must also provide a fast means of preemption. When a new burst is invoked, a background application likely holds the resources required to execute the burst. Execution of the burst is delayed until the resources can be preempted from the background application. Existing techniques using Linux cpusets can allocate or preempt a CPU core in around 30 microseconds [14]. However, granular computing infrastructure will require even lower preemption latencies to support the shortest bursts.

\subsection{Persistence}

Most applications, whether granular or not, expect their results to be persistent: the results should not be lost except under extreme (and rare) conditions. Persistence is particularly challenging for granular applications because of the small time intervals involved. For example, suppose persistence is implemented by executing each task as a transaction whose results are committed to durable storage before the task completes. Unfortunately, the latency of today's durable storage devices (disk and flash) is far too high for this approach to be viable with the small task durations that we envision.

One approach to achieve low latency persistence is to simply use a faster storage medium. Emerging nonvolatile memories, such as 3D XPoint, offer the possibility of dramatically lower write latencies compared to flash memory, perhaps as low as one microsecond or less. At this latency, it may be possible for each task to persist its results, even for the smallest tasks we envision in granular computing.

Another possible approach is to enforce persistence only occasionally, when a collection of tasks reaches a well-defined state. This allows the cost of persistence to be amortized across multiple tasks. Supporting this style of persistence would require mechanisms analogous to resilient distributed datasets in Spark [18]. With this approach, results are only occasionally made persistent, but the system retains enough persistent data that it can roll back to persistent data and recompute any lost information after a crash. One of the challenges will be to identify appropriate persistence points; this will be less obvious for granular computing than it is for Spark, and may require assistance from applications. 


\section{Conclusion}

Granular computing is currently stuck in a "no-chicken-noegg” cycle: no-one builds granular applications because our current infrastructure cannot support them, but there is no incentive to improve the infrastructure because there are no applications. In order to break the cycle, someone must create the infrastructure even though there are no applications, and then hope that the applications will follow. Since it is less likely for industry to create a product with no market, there is an opportunity for the research community to take the lead and break the cycle. If we do this, not only will the process lead to interesting research that pushes the boundaries of latency and scale, but the results could enable exciting new classes of applications.

\section{Acknowledgments}

We would like to thank Seo Jin Park, Heymian Wong, and our anonymous reviewers for their comments, feedback, and help improving this paper. We would also like to thank Yilong Li for the early discussions that helped clarify many of the ideas. This work was supported by the industrial affiliates of the Stanford Platform Laboratory.

\section{References}

[1] Amazon Web Services, Inc. 2019. AWS Lambda. https://aws.amazon com/lambda.

[2] Luiz Barroso, Mike Marty, David Patterson, and Parthasarathy Ranganathan. 2017. Attack of the Killer Microseconds. Commun. ACM 60, 4 (March 2017), 48-54. https://doi.org/10.1145/3015146

[3] Michael Dalton, David Schultz, Jacob Adriaens, Ahsan Arefin, Anshuman Gupta, Brian Fahs, Dima Rubinstein, Enrique Cauich Zermeno, Erik Rubow, James Alexander Docauer, Jesse Alpert, Jing Ai, Jon Olson, Kevin DeCabooter, Marc de Kruijf, Nan Hua, Nathan Lewis, Nikhil Kasinadhuni, Riccardo Crepaldi, Srinivas Krishnan, Subbaiah Venkata, Yossi Richter, Uday Naik, and Amin Vahdat. 2018. Andromeda: Performance, Isolation, and Velocity at Scale in Cloud Network Virtualization. In 15th USENIX Symposium on Networked Systems Design and Implementation (NSDI 18). USENIX Association, Renton, WA, 373-387. https://www.usenix.org/conference/nsdi18/presentation/dalton

[4] Jeffrey Dean and Luiz André Barroso. 2013. The Tail at Scale. Commun. ACM 56, 2 (Feb. 2013), 74-80. https://doi.org/10.1145/2408776.2408794

[5] Jeffrey Dean and Sanjay Ghemawat. 2008. MapReduce: Simplified Data Processing on Large Clusters. Commun. ACM 51 (January 2008), 107-113. Issue 1. https://doi.org/10.1145/1327452.1327492

[6] Flavio P Junqueira, Benjamin C Reed, and Marco Serafini. 2011. Zab: High-performance broadcast for primary-backup systems. In 2011 IEEE/IFIP 41st International Conference on Dependable Systems Networks (DSN). IEEE Computer Society, Los Alamitos, CA, 245-256. https: //doi.org/10.1109/DSN.2011.5958223

[7] Jay Kreps, Neha Narkhede, and Jun Rao. 2011. Kafka: A Distributed Messaging System for Log Processing. In Proceedings of the 6th International Workshop on Networking Meets Databases (NetDB 2011). ACM, New York, NY, USA, 7 pages.

[8] Grzegorz Malewicz, Matthew H. Austern, Aart J.C Bik, James C. Dehnert, Ilan Horn, Naty Leiser, and Grzegorz Czajkowski. 2010 Pregel: A System for Large-scale Graph Processing. In Proceedings of the 2010 ACM SIGMOD International Conference on Management of Data (SIGMOD '10). ACM, New York, NY, USA, 135-146. https: //doi.org/10.1145/1807167.1807184
[9] Microsoft. 2019. Microsoft Azure Functions. https://azure.microsoft. com/en-us/services/functions/.

[10] Amy Ousterhout, Joshua Fried, Jonathan Behrens, Adam Belay, and Hari Balakrishnan. 2019. Shenango: Achieving High CPU Efficiency for Latency-sensitive Datacenter Workloads. In 16th USENIX Symposium on Networked Systems Design and Implementation (NSDI 19). USENIX Association, Boston, MA, 361-378. https://www.usenix.org/ conference/nsdi19/presentation/ousterhout

[11] Kay Ousterhout, Aurojit Panda, Joshua Rosen, Shivaram Venkataraman, Reynold Xin, Sylvia Ratnasamy, Scott Shenker, and Ion Stoica. 2013. The Case for Tiny Tasks in Compute Clusters. In Presented as part of the 14th Workshop on Hot Topics in Operating Systems. USENIX, Santa Ana Pueblo, NM, 6 pages. https://www.usenix.org/conference/ hotos13/case-tiny-tasks-compute-clusters

[12] Ben Pfaff, Justin Pettit, Teemu Koponen, Ethan Jackson, Andy Zhou, Jarno Rajahalme, Jesse Gross, Alex Wang, Joe Stringer, Pravin Shelar, Keith Amidon, and Martin Casado. 2015. The Design and Implementation of Open vSwitch. In 12th USENIX Symposium on Networked Systems Design and Implementation (NSDI 15). USENIX Association, Oakland, CA, 117-130. https://www.usenix.org/conference/nsdi15/ technical-sessions/presentation/pfaff

[13] Pivotal Software, Inc. 2019. RabbitMQ - Messaging that Just Sorks. http://www.rabbitmq.com/.

[14] Henry Qin, Qian Li, Jacqueline Speiser, Peter Kraft, and John Ousterhout. 2018. Arachne: Core-Aware Thread Management. In 13th USENIX Symposium on Operating Systems Design and Implementation (OSDI 18). USENIX Association, Carlsbad, CA, 145-160. https: //www.usenix.org/conference/osdi18/presentation/qin

[15] Marc Snir, Steve Otto, Steven Huss-Lederman, David Walker, and Jack Dongarra. 1998. MPI-The Complete Reference, Volume 1: The MPI Core (2nd. (revised) ed.). MIT Press, Cambridge, MA, USA.

[16] The Apache Software Foundation. 2019. Apache ActiveMQ. http: //activemq.apache.org/.

[17] The Kubernetes Authors. 2019. Production-Grade Container Orchestration - Kubernetes. https://kubernetes.io/.

[18] Matei Zaharia, Reynold S. Xin, Patrick Wendell, Tathagata Das, Michael Armbrust, Ankur Dave, Xiangrui Meng, Josh Rosen, Shivaram Venkataraman, Michael J. Franklin, Ali Ghodsi, Joseph Gonzalez, Scott Shenker, and Ion Stoica. 2016. Apache Spark: A Unified Engine for Big Data Processing. Commun. ACM 59, 11 (Oct. 2016), 56-65. https://doi.org/10.1145/2934664 